\title{
Anatomical Particularities of the Cecum in Rabbits and Chinchillas
}

\author{
Florin STAN
}

Department of Comparative Anatomy, Faculty of Veterinary Medicine Cluj - Napoca, University of Agricultural Sciences and Veterinary Medicine, Cluj - Napoca, 3-5, Mănăștur Street, 400372, Romania, Corresponding author, e-mail: flodvm@yahoo.com

Bulletin UASVM Veterinary Medicine 71(2) / 2014,

Print ISSN 1843-5270; Electronic ISSN 1843-5378

DOI:10.15835/buasvmcn-vm: 10587

\begin{abstract}
Morphological particularities of the digestive tract are insufficiently studied; more emphasis is put on digestive physiology. Due to the major importance of the cecum in the digestive strategy of rabbits and chinchillas, we consider appropriate a detailed and comparative anatomical description of this organ. Ten rabbits and ten chinchillas were studied using gross dissection. Following dissection, using routine anatomical techniques, the organs were photographed, measured and recorded. All phases of the study were conducted in accordance to the current regulations. In rabbits, the cecum occupies the biggest part of the abdominal cavity compared with chinchillas in which the cecum was located on the left of the median plane. Particularly, in rabbits we noticed a spiral arrangement of the cecum, between the base and body being placed the proximal loop of ascending colon and the ileum. These three anatomical segments form the ileo-ceco-colic complex, a distinct anatomical entity in rabbits. In chinchillas the cecum consists of two segments, the first having a folded appearance and the second a tubular appearance. The ileo-ceco-colic junction in rabbits was obvious, compared to chinchillas. An ileocecal valve and another ceco-colic one which directs digesta were identified in both species. Compared to chinchillas, rabbits show two specific anatomical structures: sacculus rotundus located at the junction of the ileum with the cecum and vermiform appendix in the cecum extension.
\end{abstract}

Keywords: anatomy, rabbit, chinchilla, cecum, digestive tract

\section{INTRODUCTION}

Rabbit and chinchillas are true non ruminant herbivores with a particular type of digestion, so called hind gut fermentation (Holtenius and Björnhag, 1985: Davies and Davies 2003; Sakaguchi 2003). However, the species belonging of the Rodent and Lagomorphs have great noticeable differences in their intestinal anatomy, particularly in cecum anatomy (Snipes 1981, 1982a, 1982b,Kotze et al., 2006,2010 ). The cecum is usually described as first part of the large intestine, as a blind-ending tube between the ileum and ascendant colon (Barone 1997). Communication with the ileum is made through the ileo-cecal orifice (ostiumileale) and with the colon through the ceco-colic orifice (ostiumcaecocolicum). In ruminants, horse and in carnivores the cecum lies in the right side of abdominal cavity while in pigs is located in the left side. Also, there are differences regarding the shape of the cecum; from spiral (dog) to cylindrical (ruminants, pigs) blind-ending sac or like a coma (cat). Relative to other non ruminant herbivores such as horse, the rabbits and chinchillas digestive tube has an increased development to provide the nutrient requirements and increases the efficiency of utilization of fibrous nutrients (Ebino 1993; Boellinger 2007; Kohles 2014). The aim of this study was to provide a detailed morphological description of the cecum in rabbit and chinchilla in order to highlight the main similarities and differences between the two species.

\section{MATERIALS AND METHODS}

Ten rabbits and ten chinchillas of varying weights and age were used. The animals provide from particular farms and were maintained in 
proper condition after requisition. The procedural rules were followed in accordance with EU Directive 2010/63 of the European Parliament and of the Council on the protection of animals used for scientific purposes. Euthanasia was made by an overdose of isoflurane (AErrane, Baxter, USA) in all subjects. Abdominal cavity was opened by a midline incision of abdominal wall and all components were observed in situ, their connective elements were noted and photographed. The entire gastro intestinal tract was removed and after dissection of all peritoneal attachments, it was carefully distended. The length of each component was measured. Terms were used in agreement with the NAV (Nomina AnatomicaVeterinaria) 2012.

\section{RESULTS AND DISCUSSION}

In rabbit the cecum was extremely voluminous and occupied almost the entire ventral part of the abdominal cavity (Fig. 1).

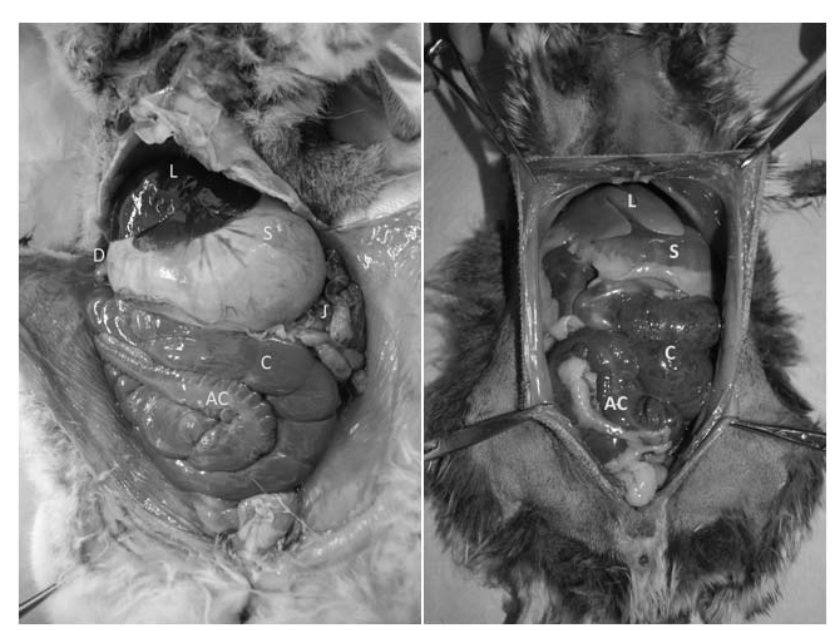

Fig. 1. Comparative view "in situ" of cecum in rabbit left and chinchilla - right, at the opening of abdominal cavity. C-cecum; L-liver; D-duodenum; S-stomach; J-jejunum; AC-ascendant colon

The cecum has a spiral shape, coiled on itself once and a half. The centre of this spiral is occupied by the base of the organ coiled, continuing on the external part with the body of the cecum. Rabbit's cecum was like a blind sac with a particular disposition, in three sections. The first section begins in umbilical region, passes cranially and slightly on the right side of abdominal cavity. Then, the second segment flexes caudally and to the right flank of abdominal cavity, parallel to the first fold, near to the pelvic cavity. The third fold runs cranially along the ventral left flank being parallel to the other segments, near to the stomach proximity and separated from the first segment by ascendant colon (Fig.2).

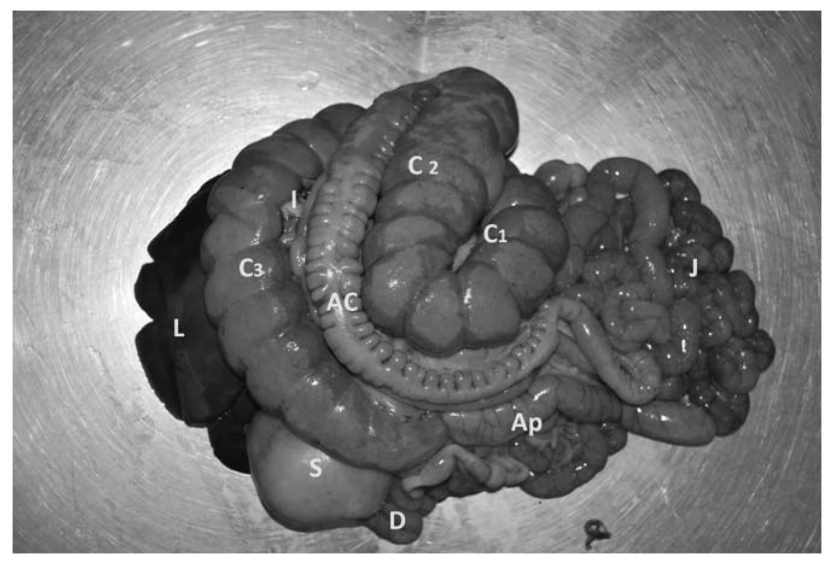

Fig.2. The ileo-ceco-colic complex in rabbit. The proximal ansa of ascending colon (AC) and the ileum (I) are disposed between the second (C2) and the third gyrus (C3) of the cecum. C1-the first gyrus ; L-liver ;

J-jejunum ; Ap-vermiform appendix ; S-stomach ; D-duodenum.

In all rabbits, at the opening of the abdominal cavity, an obvious cecocolic ampulla it was noted. A long fold extends in spiral from the cecocolic ampulla at the junction with the colon, along these first three segments, with 20-30 turns. The walls of cecum segments were thin, translucent, having greenish-grey colour due to the cecal content. The last portion of rabbit cecum, the appendix, was narrowed compared with the first segments, 10 $12 \mathrm{~cm}$ in length, with thick light pink walls, ending on the left flank, dorsal to the first segment of the cecum (Fig.3).

The appendix in all rabbits was easily viewed as an extension of the enlarged cecum with a readily discernable boundary between the cecum and the appendix marked by a sharp change in the diameter, consistency and colour compare to the cecum. On the entire length of the cecum it has been noted the presence of an axial conjunctive structure along the cecum making the connection of numerous and regular haustra. This conjunctive structure also realise the connection of the cecum with the ileum through the ileocecal fold. The cecum and the ascendant colon were connected by the ceco-colic fold. The ileum and the proximal ansa of the ascendant colon with their disposition into the cecum segments, realise the ileocecocolic 

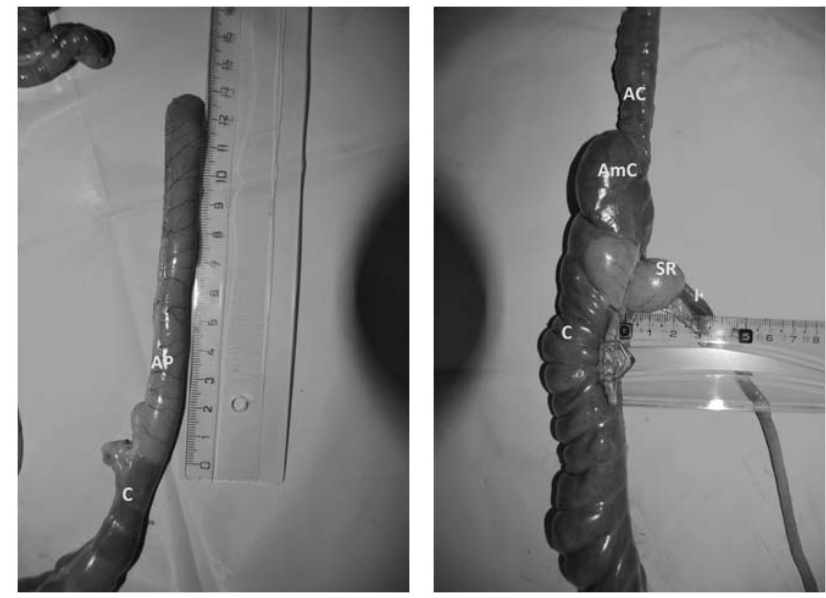

Fig. 3 The length and the shape of the vermiform appendix and sacculusrotundus in rabbit. Note the same pattern due to the lymphatic structure. Apappendix; C-cecum; SR-sacculusrotundus; I-ileum; AmC-ampulla coli; AC-ascendant colon

complex (Fig.2). In right flank the cecum was in relation with the flotant duodenum, cranial the cecum body was in relation with the stomach through the great omentum. The appendix run caudally and crosses in oblique direction the lumbar region.

At the opening of the rabbit cecum it was noted the presence of numerous longitudinal folds which maintained the external feature of haustras. The opening of ileum into the cecum was made by a simple orifice placed in the middle of lymphatic clusters which were derived from sacculus rotundus (Fig. 4). We don't observe an ileal papilla in no subject. Immediately on the periphery the ileo-cecal orifice it was observed a small circular fold which delimited the ceco-colic orifice. On the opposite wall of the ileal opening a spiral fold began with spiral distribution on the entire length of the cecum up to vermiform appendix (Fig. 4).

In chinchillas, the cecum was located on the left side of the median plane, in the ventral third of abdominal cavity and presents two different segments (Fig.1, Fig.5). The first segment was coiled having sacculations and the second segment was tubular, slightly pleated.

The sacullations of the first segment appears foliated connected by a relative large conjunctive axis which makes a round foliated appearance of the first segment of the chinchilla's cecum. The second segment corresponding to the body of thececum has fewer but larger haustras (Fig. 5).
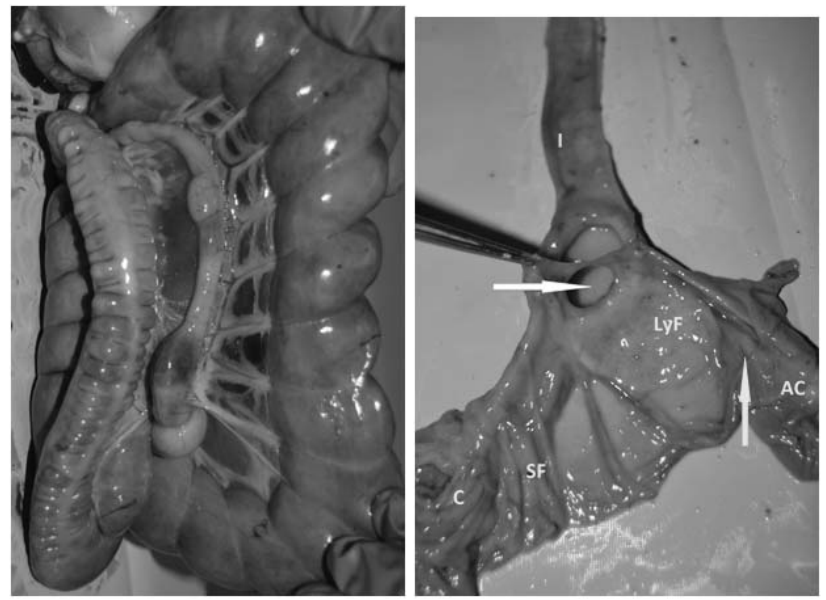

Fig. 4 The ileo-cecal fold (red arrow) and the ileo-colic fold (blue arrow) in rabbit-left. The lymphatic follicles (LyF) surround the ileocecal orifice (white arrow) and the conic shape of the ascendant colon (AC) exit from cecum (C) (yellow arrow). Note the presence of the spiral fold (SF)-right.

The ileon enter into the cecum in the first segment corresponding to the base of the organ, proximal to the ceco-colic junction. In all subjects there was only a small constriction and an obvious narrowing of the cecum which makes the transition from the cecum to the proximal ansa of the colon. Also, the colon presents numerous haustra being connected with the cecum by ceco-colic fold. The base of the cecum was connected with the transverse segment of the duodenum by ceco-duodenal fold (Fig. 5).

The cecum body and the ileum were connected by a small ileo-cecal fold. At the cecum opening the walls appears smooth macroscopically
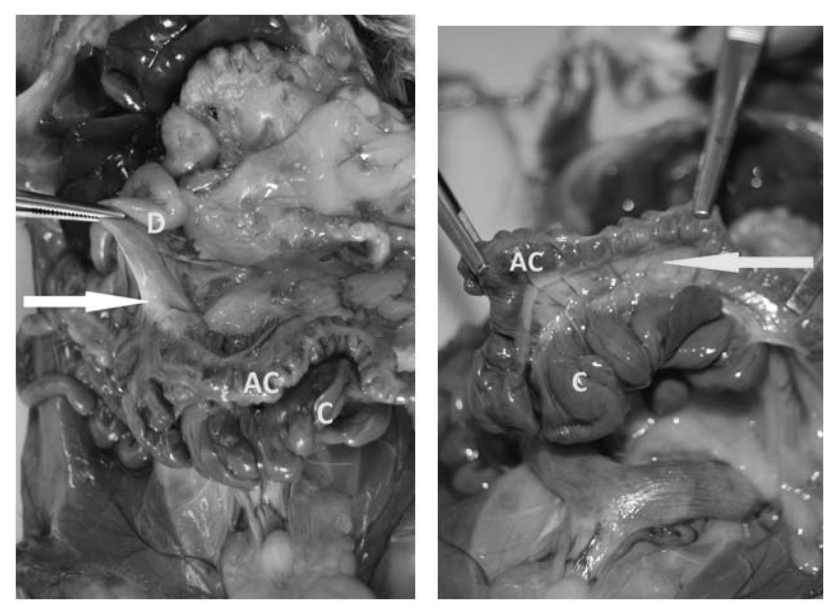

Fig. 5. The cecum of chinchilla with pleated sacculations. Note the duodeno-cecal fold (left- white arrow) and the ceco-colic fold (right- yellow arrow).Dduodenum; AC-ascendant colon; C-cecum. 
except of a few numbers of folds and depressions corresponding of haustra delimitations. The only visible differentiated structures were on the level of ileum entry into the cecum and colon exit. The ileon opening was simple, marked by a small circular structure and an obvious chance of wall colour (Fig.6).
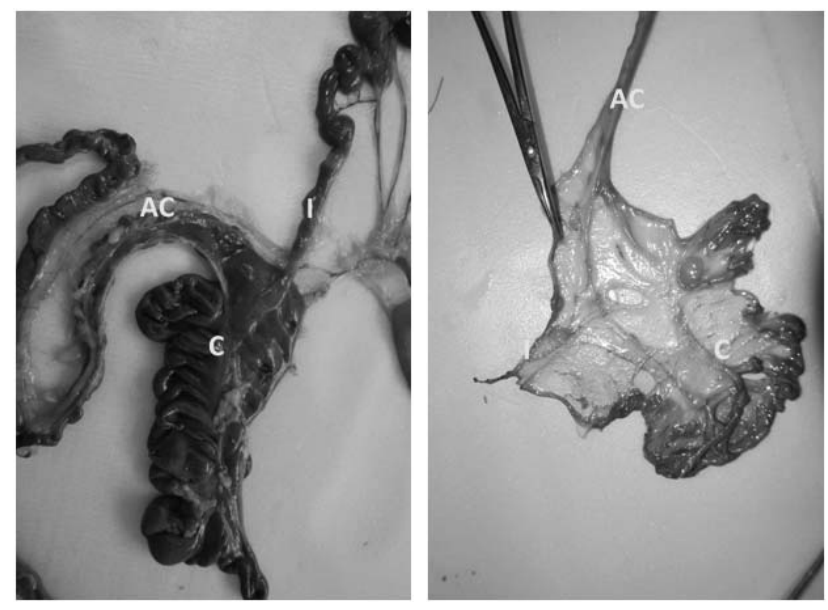

Fig.6. Ileo-ceco-colic junction in chinchilla-left and the simple layout of the cecum cavity-right, with the absence of obvious ridges and folds. C-cecum; ACascendant colon; I-ileum

Also we noted a small circumvented lip protruding into the cecum ampulla. The colon exit was marked only by a conspicuous change in calibre without changing of colour, rather as a conical passage between the cecum and the proximal ascendant ansa of the colon. On the cecum body it was visualized only a fold corresponding to the mesenterial insertion. The chinchilla's cecum showed no appendix.

The morphology of gastrointestinal tract (GIT) is relatively homogenous among different species of the same order, and the development of different parts of the GIT reflects adaptations of their habitat and diet (Stan et al., 2014). Moreover, the digestive anatomy of rabbits and chinchillas has not been completely described. Morphological description of digestive system is well represented in humans and large animals, but in small animals there are a few data in scientific literature regarding each component (Stan, 2013; Irimescu et al., 2014).

The topography of intestine with a voluminous, haustrated cecum was common in rabbit and chinchilla. In rabbit the cecum occupied almost the entire ventral part of the abdominal cavity compare to the chinchilla in which the cecum was located on the left side of the median plane. This pattern is recognised as a common feature in many rodents (Kotze et al., 2010; Perrin and Curtis 1980; Perez et al., 2008).

In the rabbit (Oryctolagus cuniculus) the ileum, cecum and a part of the ascending colon are coiled together forming a spiral with one and a half loops (Barrone, 1997) making an anatomical and physiological entity: the ileo-caeco-colic complex.

In the chinchilla (Chinchilla laniger) the ileon, cecum and the ascending colon were separated. This feature was also reported in another's rodents hindgut fermenters as nutria (Myocastor coypus) (Perez et al., 2008) capybara (Hydrochoerus Hydrochaeris) (Vasquez et al., 2012), tucu-tucu (Ctenomys pearsoni) (Perez et al., 2009). Compare to the laboratory mouse (Mus musculus) and rat (Rattus rattus)in which Snipes (1981) has described the existence of an uncoiled cecum and no haustra, in rabbit and chinchilla the presence of a voluminous saculated cecum is a common feature in both species.

Regarding the muscular tenia which were mentioned in the nutria (Perez, 2008), persian squirrel (Sciurus anomalus) (Sadeghinezhad et al., 2012), guinea pig (Cavia porecellus) (Quesenberry, and Carpenter, 2012) in the rabbit and chinchilla we describe only a conjunctive structure (axis), which being shorter than the cecum length create saccular outpunchings-haustra. Our findings are similar with Barone description (1997). In gerbils (Meriones unguiculatus) Snipes (1981) described only a few slight sacculations and the presence of poorly developed taeniae.

The cecum of lagomorphs and rodents has been divided into three distinct anatomical segments: ampulla ceci (the base of the cecum), corpus ceci and apex ceci (Perrin and Curtis 1980; Snipes 1981, 1982a,b; Perez 2005, 2007, 2008). In the rabbit we recognized the same segments but the chinchilla lacks the vermiform appendix. Also, the sacculus rotundus, the last segment of the rabbit ileum that is markedly extended in the rabbit (Barrone 1997; Stan et al., 2013) is absent in chinchilla (Langer 2002). Another particular feature of the rabbit GIT is the presence of the vermiform appendix (Barrone 1997), easily viewed as an extension of its already enlarged cecum, with a obvious discernable boundary between the cecum and the appendix marked 
by a sharp change in the diameter of the bowel, the change of the wall colour and thickness. This feature was reported by the Snipes (1982b) too.

The appendix in mammals is mentioned in humans, lagomorphs and some rodents sharing the same ontogenetic origin and high lymphoid concentration (Smith et al., 2009). In humans the role of this GALT (gut associated lymphoid tissue) is to preserve the commensal bacteria which can recolonize the colon in diseases in which these bacteria are lost (Bollinger et al., 2007). The immunologic function is supported by immune molecules such as IgA and mucin which support growth of bacterial biofilms on the epithelium of the proximal colon both in humans and in rabbits (Smith et al., 2009).

Thus it can be hypothesized that the immune function carried out by the appendix in rabbit, humans and some rodents is analogous to the immune functions carried out by the terminal portion of the cecum in animals lackingan appendix, like the chinchilla, subjects of this research. In a morpho-histological study of the digestive tract of chinchilla, (Calamar et al.,2014) have described the presence of massive development of lymphatic follicles located into submucosa layer of the chinchilla's cecum. Phisiologicaly, the vermiform appendix in rabbits is involved in bicarbonate secretion in order to buffer the cecal acidity and in water secretion to form the semifluid cecal paste (Davies et al., 2003; Kohles, 2014).

Regarding the internal structure, the presence of the spiral fold into the rabbit cecum is a particular feature and is not present in chinchilla. From a morphological point of view, the spiral fold whit its disposition and muscular core (Snipes 1978) offer a physiologically explanation of functional specialization of this organ in rabbits. Considering the coprophagy practiced by the rabbit, the spiral fold channelling the intestinal content into the corpus ceci and into the ampulla coli and practically increasing the absorption surface of the mucosa. On the other hand it's muscular core, as was assessed on scanning electron microscopy (Snipes, 1978) aiding in the contraction forces and simultaneously move the cecal content along performed ridges. Physiological research have demonstrated both descending and ascending contraction of the cecum in order to separate fluids from solid particles and maintained a longer time the nutrients which is essential for fermentation process (Franz et al., 2010).

In addition, the antiperistaltic waves from colon to the cecum are apparently a unique colonic separation mechanism (CSM) found both in rabbit and chinchilla. But, as true is the fact that in rabbits CSM is different from that in the chinchilla. Franz (2010) shown that in lagomorphs the "wash-back" CSM is more efficient in extracting bacterial matter from the colonic digesta compare to "mucus-trap" CSM found in rodents.

The ileocecocolic junction was clearly marked in the rabbit by presence of two folds located laterally of the ileocecal orifice and a compact agglomeration of lymphatic follicles surrounding the ileocecal orifice. This feature was no observed in chinchillas. About the cecocolic junction, the colon enters into the cecum as a wide conical opening without an apparent sphincter structure in both species. As a constant finding is the entry of the ileum and the exit of the colon from the ceci ampulla in close proximity. This is in accordance with the results of another researcher (Snipes, 1978; Franz et al., 2010; Calamar et al., 2014).

Regarding the connection elements, in rabbit we describe the presence of cecocolic fold extended from the cecum to the proximal loop of ascending colon and the ileocecal fold being fixed between the cecum and the ileum ending at the apex of the vermiform appendix. Perez et al., 2005 mentioned the existence of a small narrow fossa in the left sheet of the mesentery between the vermiform appendix and the distal loop of ascending colon, feature that we do not observed. In chinchilla, the ileum was attached to the ececum by the ileocecal fold, to the transverse duodenum by a small and narrow fold and to the proximal colon by the cececolic fold, similar to the report of Perez et al., 2011.

The presence of duodeno-cecal fold, derived from peritoneum was mentioned only by de Castro (2010), in chinchillas, compare to the Perez (2008), who reports the presence of a duodenocecal fold between the bases of the cecum, a short proximal part of the ascending colon - belonging to the proximal ansa - and the descendant duodenum.

Small animals need more much energy and nutrients so the smaller the animal, the greater are the energy requirements with a consequent demand to ingest more food per body mass unit. 
Thus, smaller herbivorous mammals like rabbits and chinchillas, so called hind gut fermenters, more specific cecum fermenters, have a large cecum, larger than the remaining large intestine. The cecum provides an anaerobic fermentation chamber for organisms such as Bacteroides spp. and various another organisms, which breaks down ammonia, urea, proteins and enzymes from the small intestine and cellulose (Sakaguchi, 2003).

The cecum fermentation, which results in multiplication of bacteria, seems to contribute to the efficient utilization of their food through coprophagy, both in rabbit and chinchilla. The coprophagy, more correctly the cecotrophy, is supported by specific functions of the cecum and the colon. Cecotrophy ensures that protein synthesised by bacteria growing in the distal fermentation chambers, the cecum and the colon is not lost via defecation, but reingested as soft pellets. Although modern diets mean that cecotrophy is unnecessary for survival; this feature is an innate behaviour stimulated by anal reflex (Ebino, 1993).

\section{CONCLUSION}

This research provides a comprehensive anatomical features and accurate description of the cecum in rabbit and chinchilla.

Cecum morphology showed the same characteristic regarding the presence of a voluminous, sacullated pattern, both in rabbit and chinchilla.

The differences are related to the topography, the presence of a well developed vermiform appendix in rabbit which is one of the particular features of this specie alongside the presence of sacculus rotundus. Also the internal morphology differs in rabbit and chinchilla by presence of the spiral fold in rabbit.

The unique morphological features of the digestive system in both species are the prerequisite of the physiological hind gut fermentation and offer the explanation of digestive strategy of coprophagy in rabbits and chinchillas.

\section{REFERENCE}

1. Barone R. Anatomie comparée des mammifères domestiques. 3 ed.Paris: VigotFréres,(1997). (vol. 3)

2. Bollinger RB, Barbas AS, Bush EL, Lin SS, ParkerW, (2007). Biofilms in the large bowel suggest an apparent function of the human vermiform appendix. J. Theor. Biol. 249: 826-831

3. Călămar CD, Pătruică Silvia., Dumitrescu Gabi, Bura M, Bănățean - Dunea, I, Marioara Nicula (2009) Morphohistological study of the digestive tract and the annex glands of Chinchilla laniger Scientific Papers Animal Sciences and Biotechnologies, 47 (1): 269-274.

4. Castro T, Dummer R, RickesE, Pereira M. (2010)..Aspectos morfológicos, morfométricos e topográficos do aparelho digestório de Chinchilla lanigera. Braz. J. Vet. Res. Anim. Sci. 47(1),86-94.

5. Davies RR, Davies JA,(2003). Rabbit gastrointestinal physiology. Vet. Clin.North Am Exot. Anim. Pract., 6, p. 139-153.

6. Ebino KY. (1993). Studies on coprophagy in experimental animals.Jikken Dobutsu. 42:1-9.

7. Franz, R., Kreuzer, M., Hummel, J., Hatt, J.-M.andClauss, M. (2011), Intake, selection, digesta retention, digestion and gut fill of two coprophageous species, rabbits (Oryctolagus cuniculus) and guinea pigs (Cavia porcellus), on a hay-only diet. Journal of Animal Physiology and Animal Nutrition, 95: 564-570

8. Holtenius K, Björnhag G, (1985). The colonic separation mechanism in the guinea pig (Cavia porcellus) and the chinchilla (Chinchilla laniger). Comparative Biochemistry and Physiology 82, 537-542.

9. Irimescu Irina, Adriana Chende, Ghiurco F, Damian A., (2014). Anatomical Study of the Cerebral Hemispheres in the Chinchilla (Chinchilla laniger), Bulletin UASVM Veterinary Medicine 71 (1), 130-136

10. Katherine Quesenberry, Carpenter JW,. (2012). Ferrets, Rabbits and Rodents: Clinical Medicine and Surgery 3ed ed. ISBN10: 1416066217

11. Kohles M. (2014). Gastrointestinal anatomy and physiology of select exotic companion mammals.VetClin North Am Exot Anim Pract.17(2):165-78.

12. Kotze SH, Merwe MJ O' Riain (2006).The topography and gross anatomy of the gastrointestinal tract of the cape dune mole-rat (Bathyergus suillus).Anat. Histol.Embryol., 35(4):259-264.

13. Kotze SH, Van Der Merwe, EL, Bennett, NC. O'Riain, MJ, (2010). The comparative anatomy of the abdominal gastrointestinal tract of six species of African mole-rats (Rodentia, Bathyergidae). J. Morphol., 271:50-60

14. Langer $P$, ( 2002). The digestive tract and life history ofsmall mammals. Mammal Rev, 32: p. 107-31.

15. NAV, 2012,International Committee on Veterinary Gross Anatomical Nomenclature, Fifth Edition (revised version), (ICVGAN).

16. Pérez W, M Lima, A. Bielli, (2008). Gross anatomy of the intestine and its mesentery in the nutria (Myocastor coypus). Folia Morphol., 67: 286-291.

17. Pérez W, M. Lima, A. Machado G. Izquierdo,(2009). Gross anatomy of the intestine and their peritoneal folds in the tucu - tucu (Ctenomys pearsoni). Braz. J. Morphol. Sci., 26:159-163. 
18. Pérez W, N. Vazquez, H. Jerbi,( 2011). Gross anatomy of the intestine and their peritoneal folds in the chinchilla (Chinchilla lanigera). J. Morphol. Sci.28: 180-183.

19. Pérez W, R. Möller, E. Martin, (2005). Peritoneal folds of the rabbit (Oryctolagus cuniculus). Anat.Histol. Embryol., 34: 167-170.

20. Pérez W, R. Möller, E. Martin. (2007). Suggested nomenclature for the cecum and ascending colon of the rabbit. Anat. Histol. Embryol., 36: 389-395.

21. Perrin MR. BA.Curtis, (1980).Comparative morphology of the digestive system of 19 species of Southern African myomorph rodents in relation to diet and evolution. S. Afr. J. Zool., 15: 22-33.

22. Sadeghinezhad J Z Tootian, GH Akbari, R Chiocchetti ,(2012),The Topography and Gross Anatomy of the Abdominal Gastrointestinal Tract of the Persian Squirrel (Sciurus anomalus)Int. j. morphol 30 (2), 524-530

23. Sakaguchi E, (2003),. Digestive strategies of small hindgut fermenters. Animal Science Journal 74, 327-337.

24. Smith H F, Fisher RE, Everett ML, Thomas AD, Randal Bollinger R. Parker W. (2009), Comparative anatomy and phylogenetic distribution of the mammalian cecal appendix. Journal of Evolutionary Biology, 22: 19841999.

25. Snipes RL 1978 Anatomy of the rabbit cecum Anatomy and Embryology 155, (1) :57-80.

26. Snipes RL, (1982a). Anatomy of the guinea-pig cecum. Anat. Embryol.165:97-111.

27. Snipes RL, 1981. Anatomy of the cecum of the laboratory mouse and rat. Anat. Embryol., 162:455-474.

28. Snipes RL, (1982b). Anatomy of the cecum of the gerbil (Meriones unguiculatus) (Mammalia,Rodentia, Cricetidae).Zoomorphology 100 (3): 189-202.

29. Stan F, (2013.) Comparative Study Of The Stomach Morphology In Rabbit And Chinchilla AgroLife Scientific Journal, Bucuresti, Romania, 2013 (2):73-78.

30. Stan F, Damian A, Gudea A, Dezdrobitu C., DeliaBob, Martonos C, Ileana Bochis, Pogana B, (2013).Comparative anatomical study of the large intestine in rabbit and chinchilla. Bulletin of UASVM Cluj-Napoca,Veterinary Medicine, Vol. 69(1-2).

31. Vazquez N, R. Senos W. Pérez, (2012). Anatomy of the gross intestine of the capybara (Hydrochoerus hydrochaeris). Am. J. Anim. Vet. Sci., 7: 92-95. 